\title{
Using a Curricular Vision to Define Entrustable Professional Activities for Medical Student Assessment
}

\author{
Karen E. Hauer, MD, Christy Boscardin, PhD, Tracy B. Fulton, PhD, Catherine Lucey, MD, \\ Sandra Oza, MD, MA, and Arianne Teherani, PhD
}

San Francisco School of Medicine, University of California, San Francisco, CA, USA.

\begin{abstract}
BACKGROUND:The new UCSF Bridges Curriculum aims to prepare students to succeed in today's health care system while simultaneously improving it. Curriculum redesign requires assessment strategies that ensure that graduates achieve competence in enduring and emerging skills for clinical practice.
\end{abstract}

AIM: To design entrustable professional activities (EPAs) for assessment in a new curriculum and gather evidence of content validity.

SETTING: University of California, San Francisco, School of Medicine.

PARTICIPANTS: Nineteen medical educators participated; 14 completed both rounds of a Delphi survey.

PROGRAM DESCRIPTION: Authors describe 5 steps for defining EPAs that encompass a curricular vision including refining the vision, defining draft EPAs, developing EPAs and assessment strategies, defining competencies and milestones, and mapping milestones to EPAs. A $\mathrm{Q}$-sort activity and Delphi survey involving local medical educators created consensus and prioritization for milestones for each EPA.

PROGRAM EVALUATION: For 4 EPAs, most milestones had content validity indices (CVIs) of at least $78 \%$. For 2 EPAs, 2 to 4 milestones did not achieve CVIs of $78 \%$.

DISCUSSION: We demonstrate a stepwise procedure for developing EPAs that capture essential physician work activities defined by a curricular vision. Structured procedures for soliciting faculty feedback and mapping milestones to EPAs provide content validity.

KEY WORDS: clinical competence; assessment; undergraduate medical education; program evaluation.

J Gen Intern Med 30(9):1344-8

DOI: $10.1007 / \mathrm{s} 11606-015-3264-\mathrm{Z}$

(c) Society of General Internal Medicine 2015

\section{INTRODUCTION}

Clinical performance assessment should occur in the context in which clinical care occurs. ${ }^{1}$ Workplace-based assessments typically address multiple competency domains and capture learners' ability to synthesize their knowledge and skills to conduct work. ${ }^{2}$ Educators have embraced competency-based

Electronic supplementary material The online version of this article (doi:10.1007/s11606-015-3264-z) contains supplementary material, which is available to authorized users.

Published online July 15, 2015 medical education (CBME) as a strategy to encourage individual developmental paths toward competence. However, meaningful implementation of CBME in the workplace has been challenging. Detailed competencies and milestones raise concerns that resulting checklists can fail to capture how learners actually perform in clinical settings. The workplace structure and pace can further impede performance assessment. ${ }^{3,4}$

Entrustable professional activities (EPAs) are a strategy to operationalize competencies and milestones in the workplace and focus supervisors and learners on key activities to be assessed. ${ }^{5,6}$ Graduate medical educators have started to implement EPAs as a framework for assessment across specialties. ${ }^{7-10}$ Undergraduate medical educators are beginning to consider how trust can inform student assessment, particularly in settings that afford longitudinal contact between students and supervisors and meaningful student roles. ${ }^{11,12}$ Nationally, the Association of American Medical Colleges (AAMC) has proposed EPAs for graduating students to ensure readiness for residency. ${ }^{13}$ However, to our knowledge, a process for developing student-level EPAs that address institutional program objectives, student competencies, and larger mandates for CBME has not been articulated. The purpose of this manuscript is (1) to describe how we used the vision for a new curriculum to develop EPAs, and (2) to outline a process that can be used by other institutions to establish content evidence $^{14}$ for EPAs that encompass their program objectives, competencies and milestones, and overall assessment plan.

\section{SETTING AND PARTICIPANTS}

The University of California, San Francisco (UCSF) is an urban public medical school. The curriculum includes two years of foundational science coursework and some clinical preceptorships, a third core clinical year, and a fourth year of clinical electives and options for concentration. The Institutional Review Board approved this project.

\section{PROGRAM DESCRIPTION}

We followed a stepwise process based upon the Standards for Educational and Psychological Testing ${ }^{14}$ to establish content validity of our EPAs. The Standards define sources of validity 
evidence that can confirm how well an assessment measures what it was designed to measure. Below, we outline the steps we employed to establish the content evidence for our EPAs. We describe the purpose of each step in the continuum of curricular and assessment change, and the result of that step. These steps can be applied in other settings to establish content evidence for new and existing EPAs at all educational levels.

\section{Step 1: Define a Curricular Vision}

We sought to frame our assessment process within our school's new curricular vision. At UCSF, this vision was defined in the context of the health care system as a whole and named the UCSF Bridges Curriculum. ${ }^{15}$ The School's leaders proposed that 21 st century physicians must actively improve the health of communities by ensuring healthcare quality, access, and innovation. To meet the needs of a diverse population in an era of increasingly complex acute and chronic disease, the physician role must be redesigned to embrace interprofessional and interdisciplinary teamwork in advancing science and care delivery; to recognize the central role of complex systems and inquiry in medicine and biomedical science; and to leverage biologic, clinical, and outcomes data to enhance patients' health. A central curricular feature is authentic workplace learning experiences in patient health and systems improvement.

With this in mind, the School convened a committee to envision the ideal physician graduate. This Vision committee comprised 40 faculty and students known as visionary thinkers and experts in their fields from university hospitals and affiliated community-based practices. The group was charged with characterizing the extent and causes of persistent gaps in quality, safety, equity, evidence, and patientcenteredness in today's healthcare systems. They used this information to define the roles and competencies of physicians ideally suited to contribute to new models of healthcare and biomedical discovery.

\section{Step 2: Define EPAs Based on the Curricular Vision}

Next, we defined the constructs we aimed to assess with our EPAs. A Steering committee comprised of deans and teaching faculty with educational leadership roles, all familiar with EPAs, listed over 20 activities that characterize the previously defined roles and competencies of 21 st century physicians. The Steering committee next described the essential functions necessary to perform each activity successfully. Because the curriculum vision focused on physicians in practice, so did the EPAs. We were mindful that students would first need to achieve foundational knowledge and skills, and would perform new EPAs at a lower level of independence than practicing physicians, according to Ten Cate's ranking of entrustment. ${ }^{16}$

To authenticate this list of activities and translate it to candidate EPAs, 3 groups were targeted for feedback. First, the Vision committee (see step 1) provided feedback about the representativeness compared with envisioned physician activities and healthcare systems needs. The initial list was too long, redundant, and excluded some essential physician behaviors. Feedback clarified that behaviors on the list fell into two categories: enduring physician skills: i.e. doctor-patient communication skills and clinical reasoning, and emerging physician skills: i.e. functioning effectively within complex systems and working collaboratively in teams. Second, a Curriculum Re-design committee focused on foundational science and early clinical skills provided comments with emphasis on the importance of inquiry in advancing knowledge. Finally, at multiple points during the design process, we sought feedback from local assessment and EPA experts about whether the EPA wording would capture actual physician work.

Based on this feedback, the Steering committee defined 6 essential EPAs (Table 1) that they felt captured the range of physician work operationalizing these roles. Each EPA is characterized by enduring and emerging physician skills. The educational community approved this final list at a Vision committee meeting and larger educational retreat in Spring 2014.

\section{Step 3: Develop EPAs and Assessment Strategies}

The School's leadership charged a Student Assessment Committee to develop an assessment blueprint ${ }^{14}$ that defined each EPA, skills essential to successful completion, and student assessment strategies. This group also recommended assessment tools based on existing assessments as well as new strategies described in the assessment literature. ${ }^{16}$ They mapped the EPAs to the AAMC EPAs to ensure that key national recommendations were represented.

\section{Step 4: Define Competencies and Milestones}

Starting with the School's previously defined competencies and milestones, (http://meded.ucsf.edu/ume/md-competencies) a subcommittee of the Assessment Committee led a process to produce "graduation milestones" that reflect Bridges curriculum aims. The School's competency directors, the Assessment Committee, directors of the foundational science and early clinical skills committees, and other content experts critically reviewed drafts.

To ensure that no critical content was missing, we directly compared draft graduation milestones to AAMC general physician competencies. ${ }^{17}$ This resulted in the decision to add a new competency domain, Interprofessional Collaboration, to the School's existing 6, and also prompted creation of several new milestones related to personal and professional development. Content experts compared draft graduation milestones to intern milestones in 4 large specialties: family medicine, internal medicine, pediatrics, and psychiatry. This crosscheck identified an area not represented in the draft graduation 
Table 1 Six EPAs for Medical Students and Selected Example Essential Skills for Performing Each EPA

EPA 1: Evaluate and care for a patient with an acute complaint

- Demonstrates knowledge of pathophysiology, risk factors including psychosocial/cultural determinants of health

- Demonstrates H \& P skills, clinical problem solving, communicating with patients

- Utilizes medical literature appropriately to answer patient care questions

- Uses technology (point of care testing, point of care decision support, practice guidelines)

- Uses technology-enhanced monitoring

- Incorporates consideration of costs into management plans

- Demonstrates interprofessional collaboration

- Engages in effective crisis communication with team members while responding to an acutely deteriorating patient

EPA 2: Evaluate and care for a patient with a chronic medical problem

- Demonstrates knowledge of pathophysiology, disease course, risk factors, prognosis

- Demonstrates knowledge of behavioral health traits that factor into chronic disease

- Engages in patient education, shared decision making

- Formulates clinical questions regarding patient care

- Generates and enacts a plan for the transition to the next point of care

- Incorporates consideration of cost benefit and cost effectiveness into management plans

- Uses technology such as: risk stratification tool, electronic medical record to track and document care, technology-enhanced monitoring

- Identifies and engages appropriate interprofessional colleagues to work with for optimal disease management.

EPA 3: Identify the need for, perform, and interpret the results or outcome of a medical procedure

- Demonstrates knowledge of the indications for diagnostic tests and procedures

- Discusses the risks and benefits of tests and procedures

- Demonstrates knowledge of test characteristics, appropriate settings in which to conduct procedures, common contraindications for procedures

- Interprets the findings from a test or procedure

- Participates in the informed consent discussion with patients, detailing the risks, benefits and alternatives of the procedure

- Performs procedures with supervision

- Describes critical errors in the procedure and can describe steps to prevent them

EPA 4: Conduct a preventive care visit

- Demonstrates knowledge of risk factors to patient health and pre-cursors to onset of disease

- Interprets research identifying preventative action and risk

- Individualizes the provision of preventive services according to patient preference and values

- Incorporates current guidelines into patient care

- Utilizes validated risk stratification tools (e.g. Gail model breast cancer risk assessment tool; pooled cohort estimate for cardiovascular disease risk)

EPA 5: Address a quality of care challenge to improve health care quality and value

- Works within a clinical environment to identify an important quality or safety indicator for patient care

- Demonstrates knowledge of how to conduct a systems assessment

- Demonstrates capacity to apply system-level approaches (i.e. root cause analysis or PDSA cycles) to determine the cause of medical errors, prevent medical errors, implement a change plan aimed at improving quality of care

- Identifies relevant data and metrics to assess performance

- Identifies and engages appropriate interprofessional colleagues to: conduct a data driven process improvement program to optimize performance

- Assess outcomes in a series of cases as related to quality of care

EPA 6: Evaluate and manage a health care problem for a population of patients

- Demonstrates knowledge of population/public health assessment and research methodologies

- Works with others to query care databases to monitor important patient and population outcomes

- Develops an evaluation strategy to monitor success.

- Incorporates consideration of value and cost in planning a solution

- Identifies and engages appropriate interprofessional colleagues including population health professionals and community staff/volunteers

milestones related to requesting and providing consultative care. Defining the milestones required that we envision an expected level of competence for a graduate. We made this determination with input from clinical education leaders such as clerkship directors in the School, and through the review by residency program directors to ensure alignment between expectations at graduation and early in internship. Further edits resulted in $51 \mathrm{draft}$ graduation milestones.

\section{Step 5: Map Milestones to EPAs}

We conducted two activities to map the graduation milestones to EPAs. First, 6 educators representing the foundational, clinical, and educational sciences participated in a Q-sort activity. Q-sorting is a method for building consensus on prioritization of milestones for EPAs. ${ }^{18}$ The educators worked in 2 teams to rate milestones based on importance (least [1] to most [5]) to the assessment of each EPA. The 2 teams compared results, noting overlapping or disparate milestones for each EPA. The group conducted this process for 4 EPAs in person, and due to time constraints, for 2 EPAs individually by email. Three of the 51 graduation milestones did not map to any EPA, and 4 mapped to all EPAs. An additional round of editing led to an updated set of 40 graduation milestones.

To provide additional evidence for content validity, we used these results in an online survey of local faculty experts in clinical systems, medical school clinical skills and assessment, using the Delphi technique. Twenty-four faculty were asked to rate milestones that were scored as 2 or higher in the Q-sort for each EPA; based on Q-sort results, there were 11 to 15 milestones for each EPA. The survey queried the importance of each candidate milestone for the corresponding EPA (least [1] to most [5] important). Of the 24 invited experts, 19 faculty responded to the Round 1 survey. Out of 19, 14 responded to both rounds ( $74 \%$ return rate). We calculated content validity indices $(\mathrm{CVI})^{19}$ (the percentage of respondents rating an item as important, defined as 4 or 5 on our scale) and mean importance ratings for candidate milestones for each EPA. A CVI of $78 \%$ or greater signifies evidence of consensus and validity. ${ }^{19}$ 


\section{PROGRAM EVALUATION}

Table 2 (available online) provides CVIs and mean importance ratings for representative candidate milestones for each EPA. For EPA 1, 11 of 13 milestones had CVIs higher than $78 \%$, representing standard criteria for consensus and content validity. For EPA 2, one milestone did not meet the content validity standard, with mean rating of 3.6 and low CVI score (42.8\%). For both EPA 3 (15 milestones) and EPA 4 (11 milestones), all candidate milestones were rated as meeting the content validity criteria for consensus. EPA 5 (2 milestones) and EPA 6 (4 milestones) had the most milestones with lack of consensus among faculty experts on the validity of the candidate milestones. For EPA 5, 2 milestones fell below the $78 \%$ standard for CVI. EPA 6 had the most milestones $(n=4)$ that did not reach the $78 \%$ CVI score; only $66 \%$ of the candidate milestones reached consensus.

\section{DISCUSSION}

Our experience demonstrates a stepwise, replicable procedure to provide evidence of EPA content validity in undergraduate medical education (UME). We used a curricular vision to guide the development and refinement of candidate EPAs and mapped graduation milestones to those EPAs.

The appropriate size and scope of individual EPAs is a topic actively debated in the literature. Our focus on the physician that our institution aims to produce led to EPAs similarly focused on practicing physicians. We mapped our school's EPAs to the AAMC's core EPAs for entering residency and found that the content overlapped significantly. ${ }^{13}$ Some of our school's EPAs encompass multiple AAMC EPAs, which themselves have contributed to questions about how broad or focused an individual EPA should be. ${ }^{20}$ To be useful for assessment, an EPA must be observable, replicable, and understandable to learners and supervisors. ${ }^{6,21}$ Therefore, a necessary next step in our EPA development is to specify further details for each EPA, including information about the patient, context, and learner behaviors. ${ }^{22}$ Another approach is to define smaller units of activities that nest together within larger EPAs, as has been proposed with Observable Professional Activities. ${ }^{23}$

Our approach to EPA development and mapping to milestones has limitations. This work occurred at a single institution. Educators who completed consensus-building surveys may not represent the perspectives of educators elsewhere, and may have responded differently after greater experience with the EPAs. Strengths of our approach include the consistent emphasis on operationalizing a curricular vision with broad-based faculty involvement to promote buy-in, aligning with national resources, achieving an acceptable response rate, and employing a stepwise approach that we believe is feasible and replicable. Our application of a stringent CVI cut-point provides support for our findings. Additional work is needed to provide other evidence of validity. ${ }^{14}$
We plan to pilot individual EPAs with two student groups before larger implementation. Students between their first and second year of medical school conducting curriculum development and quality improvement projects will provide insight into EPA 5. Third-year students in a year-long integrated clerkship and their longitudinal preceptors will participate in piloting EPAs 1 and 2.

In summary, guided by a curricular vision, we defined EPAs in UME and used a structured process for mapping milestones to EPAs to develop evidence of content validity. This work will serve as the basis of student assessment at our institution and inform future work on the validity of our EPAs.

Acknowledgments: The authors thank Holly Nishimura for help with data collection, and the educators who participated in the curriculum design.

Conflict of Interest: The authors declare that they do not have a conflict of interest.

Funding sources: The UCSF School of Medicine received funding from the American Medical Association as part of its Accelerating Change in Medical Education initiative.

Corresponding Author: Karen E. Hauer, MD; San Francisco School of Medicine, University of California, San Francisco, CA, USA (e-mail: karen.hauer@ucsf.edu).

\section{REFERENCES}

1. Epstein RM. Assessment in medical education. N Engl J Med. 2007;356:387-96.

2. Schuwirth LW, van der Vleuten CP. General overview of the theories used in assessment: AMEE Guide No. 57. Med Teach. 2011;33:783-97.

3. Howley LD, Wilson WG. Direct observation of students during clerkship rotations: a multiyear descriptive study. Acad Med. 2004;79:276-80.

4. Bernabeo EC, Holtman MC, Ginsburg S, Rosenbaum JR, Holmboe ES. Lost in transition: the experience and impact of frequent changes in the inpatient learning environment. Acad Med. 2011;86:591-8.

5. Carraccio C, Burke AE. Beyond competencies and milestones: adding meaning through context. J Grad Med Educ. 2010;2:419-22.

6. Ten Cate O. Trust, competence, and the supervisor's role in postgraduate training. BMJ. 2006;333:748-51.

7. Boyce P, Spratt C, Davies M, McEvoy P. Using entrustable professional activities to guide curriculum development in Psychiatry training. BMC Med Educ. 2011;11:96.

8. Bhuyan N, Miser WF, Dickson GM, et al. From family medicine milestones to entrustable professional activities (EPAs). Ann Fam Med. 2014;12:380-1.

9. Beeson MS, Warrington S, Bradford-Saffles A, Hart D. Entrustable professional activities: making sense of the emergency medicine milestones. J Emerg Med. 2014;47:441-52.

10. Hauer KE, Soni K, Cornett P, et al. Developing entrustable professional activities as the basis for assessment of competence in an internal medicine residency: a feasibility study. J Gen Intern Med. 2013;28:1110-4.

11. Hirsh DA, Holmboe ES, ten Cate O. Time to trust: longitudinal integrated clerkships and entrustable professional activities. Acad Med. 2014;89(2):201-4.

12. Chen HC, Sheu L, O'Sullivan P, Ten Cate O, Teherani A. Legitimate workplace roles and activities for early learners. Med Educ. 2014;48(2):136-45.

13. Core Entrustable Professional Activities for Entering Residency. Association of American Medical Colleges. Available at: https://members. aamc.org/eweb/upload/Core\%20EPA\%20Curriculum\%20Dev\%20Guide. pdf. Accessed October 10, 2014.

14. American Psychological Association, American Educational Research Association, and National Council on Measurement in Education. 
Standards for Educational \& Psychological Tests. American Psychological Association; 2014.

15. Lucey CR. Medical education: part of the problem and part of the solution. JAMA Intern Med. 2013;23(173):1639-43.

16. Ten Cate O, Scheele F. Viewpoint: Competency-based postgraduate training: can we bridge the gap between theory and clinical practice? Acad Med. 2007;82:542-7.

17. Englander R, Cameron T, Ballard AJ, Dodge J, Bull J, Aschenbrener CA. Toward a common taxonomy of competency domains for the health professions and competencies for physicians. Acad Med. 2013;88:1088-94.

18. Meade LB, Caverzagie KJ, Swing SR, et al. Playing with curricular milestones in the educational sandbox: $\mathrm{Q}$-sort results from an internal medicine educational collaborative. Acad Med. 2013;88:1142-8.
19. Polit DF, Beck CT, Owen SV. Is the CVI an acceptable indicator of content validity? Appraisal and recommendations. Res Nurs Health. 2007;30:45967.

20. Ten Cate O. Trusting graduates to enter residency: what does it take? J Grad Med Educ. 2014;6:7-10.

21. Lurie SJ, Mooney CJ, Lyness JM. Commentary: pitfalls in assessment of competency-based educational objectives. Acad Med. 2011;86:412-414.

22. Mulder H, Ten Cate O, Daalder R, Berkvens J. Building a competencybased workplace curriculum around entrustable professional activities: the case of physician assistant training. Med Teach. 2010;32:e453-9.

23. Warm EJ, Mathis BR, Held JD, Pai S, et al. Entrustment and mapping of observable practice activities for resident assessment. J Gen Intern Med. 2014;29:1177-82. 